\title{
Nanomaterials for Biomedical Applications: Synthesis, Characterization, and Applications
}

\author{
M. Bououdina, ${ }^{1}$ S. Rashdan, ${ }^{2}$ J. L. Bobet, ${ }^{3}$ and Y. Ichiyanagi ${ }^{4}$ \\ ${ }^{1}$ Nanotechnology Centre, University of Bahrain, P.O. Box 21038, Bahrain \\ ${ }^{2}$ Department of Chemistry, College of Science, University of Bahrain, P.O. Box 21038, Bahrain \\ ${ }^{3}$ Institut de Chimie de la Matière Condensée de Bordeaux, University Bordeaux I, CNRS-UPR 9048, France \\ ${ }^{4}$ Department of Physics, Yokohama National University, Yokohama 240-8501, Japan
}

Correspondence should be addressed to M. Bououdina; mboudina@gmail.com

Received 18 July 2013; Accepted 18 July 2013

Copyright (C) 2013 M. Bououdina et al. This is an open access article distributed under the Creative Commons Attribution License, which permits unrestricted use, distribution, and reproduction in any medium, provided the original work is properly cited.

\begin{abstract}
Nanoscience and nanotechnology and their wide applications have become spread field worldwide because nanomaterials have novel and unique properties. The properties at the nanoscale are dependent on the size, the shape, and the components. There are a variety of nanoparticle systems currently under investigation to be applied in biomedical with the emphasis on cancer therapeutics. There are a variety of nanoparticle systems currently investigated and explored for biomedical applications with some particular emphasis for cancer therapeutics; hence some precious metals (mainly gold and silver systems, $\mathrm{Au}$, and $\mathrm{Ag}$ ) and some magnetic oxides (in particular magnetite $\mathrm{Fe}_{3} \mathrm{O}_{4}$ ) received much interest including quantum dots and some of what is called "natural nanoparticles."

Also, various chemical routes have been reported for the synthesis and surface functionalisation by the attachment of specific biomolecules, optical properties, and biomedical applications of noble metal nanoparticles with various sizes, desirable shapes (nanospheres, nanodots, nanoshells, etc.), different structures, and hence tunable plasmonic properties.

This special issue is devoted to the latest techniques in nanomaterials synthesis, characterization, and biomedical applications and cancer treatment and includes broad topics such as recent development in nanomaterial synthetic methods, in particular magnetic nanoparticles, gold Nanoparticles, latest development in nanoparticle coating/core-shell, nanoparticle surface modification, role of nanomaterials
\end{abstract}

in cancer treatment, identifying the mechanism of cell penetration of the nanomaterials, and recent development in using nanomaterials in photo-killing of bacteria.

The special issue received 18 papers and after peerreviewing process only 9 papers (50\%) have been accepted. The paper presented by C. A. Zamperini et al. was devoted to the study of antifungal effect of hydroxyapatite $\mathrm{Ca}_{10}\left(\mathrm{PO}_{4}\right)_{6}(\mathrm{OH})_{2}(\mathrm{HA})$ nanorods decorated with silver (Ag) nanospheres against Candida albicans planktonic cells. Fungistatic and fungicidal effects against tested microorganisms were observed, indicating the possibility of developing new antimicrobial agents with multiple applications in biomedical field. Moreover, a significant reduction in the number of $\mathrm{CFU} / \mathrm{mL}$ was observed only at concentration of $1000 \mu \mathrm{g} / \mathrm{mL}$. The biofilm matrix acts as a barrier thus protecting the deep layers of cells. SEM images reveal alterations in cell morphology ("shriveled" cells appearance), which are proportional to the concentration of HA@Ag NPs, even though some intact cells remain for higher NPs concentration. A reaction mechanism involving $\mathrm{O}_{2}$ and $\mathrm{H}_{2} \mathrm{O}$ adsorption was proposed to justify the antifungal activity by the interaction between the structure and the defects density variation in the interfacial (HA@Ag) and interfacial (HA) region with the fungal medium. The decomposition and desorption of the final products as well as the electron/hole recombination process have an important role in fungicidal effects. 
The paper presented by Alsayed A. M. Elsherbini discusses the possibility of using magnetic NPs, for this case magnetite $\mathrm{Fe}_{3} \mathrm{O}_{4}$ suspended in glycerine solution, for hyperthermia via alternating magnetic field (AMF), in Ehrlich carcinoma cells implanted in female mice. It was found that

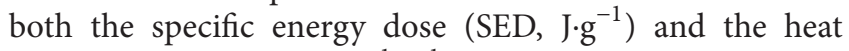
deposition rate (HDR, $\mathrm{J} \cdot \mathrm{g}^{-1} \cdot \mathrm{s}^{-1}$ ) are directly proportional to the amount (dose) of $\mathrm{Fe}_{3} \mathrm{O}_{4}$ NPs injected within tumors. The maximum temperature recorded inside the tumor subjected to $\mathrm{AMF}$ increases with increasing MNPs dose, varying in the range $40.11-48.6^{\circ} \mathrm{C}$ for $200-1000 \mu \mathrm{g}$.

C. Hara et al. investigated the adsorption of the hormone melatonin to polyethylene glycol (PEG) microspheres and its functional activity of human colostrum phagocytes. Fluorescence microscopy and flow cytometry showed that PEG microspheres had ellipsoid shapes and were easily separated from the suspension, with a size in the range of $5.8 \mu \mathrm{m}$. After adsorption of melatonin, the size was reduced to approximately $5.35 \mu \mathrm{m}$, suggesting that melatonin may bind at the same site as the marker. It is found that this microspherebased polymeric substance stimulates the functional activity of colostrum phagocytes as evidenced by the release of superoxide and intracellular calcium and thus can be used for controlled drug delivery. The superoxide anion release decreased but remained higher than that found during spontaneous release. This suggested that PEG microspheres can modify the release of melatonin while maintaining cellular activation. It is stated that the beneficial actions of melatonin are associated with its ability to remove free radicals and increase the enzymatic activity of antioxidants, as well as its immunostimulatory effects, and can stimulate cells of the immune system.

L. Ventrelli et al. presented a review paper titled "Nanoscaffolds for guided cardiac repair: the new therapeutic challenge of regenerative medicine." The authors reported and discussed in detail the most recent achievements in the field of cell therapy for myocardial infarction treatment and heart regeneration, focusing on the commonly used cell sources, traditional approaches used to delivery cells at the damaged site. Moreover, a series of novel technologies based on recent advancements of bioengineering and tissue engineering have been illustrated, as well as highlighting patches, fragments, and biomaterials. Additionally, an original strategy for cardiac repair based on magnetic nanosheets has been discussed, highlighting the tremendous potential and promises that nanoscaffolds have within the therapeutic challenge related to heart regeneration. Finally, based on the previous findings, the authors concluded that magnetic nanofilm-based approach looks really the most promising. This therapeutic philosophy implies that the injection of cell-seeded nanosheets inside the body and its guidance up to the damaged heart site can deeply improve MI therapeutic outcomes. Furthermore, the possibility of loading the nanofilm with specific growth factors or drugs makes this new bioengineering approach even more appealing and promising.

A paper titled "Adsorption of albumin on silica surfaces modified by silver and copper nanoparticles" was presented by P. Kumari and P. Majewski. Using magnetron sputtering method, $\mathrm{Cu}$ and $\mathrm{Ag}$ NPs were produced on the surface of silica slides (confirmed by that UV-V is spectra), where size, shape (spherical to random), and concentration depend on sputter time and plasma power. XPS reveals formation of metal oxides, $\mathrm{Ag}_{2} \mathrm{O}$, and $\mathrm{CuO}$. Matrix-assisted laser desorption/ionization mass spectrometry analyses of the albumin adsorption on the surface with attached Ag-NPs indicate a significant delay compared to pure silica surface; albumin ${ }^{1+}$ and albumin ${ }^{2+}$ peaks appear after $24 \mathrm{hrs}$. For the case of $\mathrm{Cu}-$ NPs, albumin ${ }^{1+}$ and albumin ${ }^{2+}$ are visible after $3 \mathrm{hrs}$ and $24 \mathrm{hrs}$, but it was noticed that the peaks are significantly weaker compared to those on pure silica and silica with AgNPs at albumin exposure for 24 hrs. This is because Cu-NPs ( 1 to $5 \mathrm{~nm}$ ) are smaller than Ag-NPs (10 to $20 \mathrm{~nm}$ ), thereby larger albumin molecules (about 8 to $10 \mathrm{~nm}$ ) are able to attach to the silica surface to some extent by bridging smaller NPs. Finally, it was concluded that if protein attachment can be prevented during that time period, as shown for surfaces coated with Ag-NPs, the chance of infection is clearly reduced.

N. Songvorawit et al. presented a study devoted to development of antibodies conjugated fluorescent dye-doped silica nanoparticles (FDS-NPs) for the rapid detection of Escherichia coli O157:H7 with glass slide method. FDS-NPs were successfully synthesized by modified sol-gel reaction via water-in-oil microemulsion method, giving spherical shape with average sizes of $47 \pm 6 \mathrm{~nm}$. High hydrophilic Rubpy dye was chosen as an inorganic fluorescent dye. SEM-EDS results show the presence of $\mathrm{Si}$ (11.31\% atomic), O $(64.43 \%$ atomic), $\mathrm{Ru}(0.15 \%$ atomic), and $\mathrm{C}(24.11 \%$ atomic). The existence of Ru confirmed that Rubpy dye was doped inside the particles. FTIR spectra of FDS-NPs confirm that the amine group was successfully attached onto particles' surface, which facilitate the bioconjugation with the target antibodies. Thus, glutaraldehyde, which is well known as a crosslinking reagent between two amino groups of protein, was used for FDS-NPs-IgGs conjugation. Results of antibodies conjugation (IgGs) with FDS-NPs show that the average amount of immobilized IgGs on amino-modified FDS-NPs increases with increasing incubation time and temperature ( 4 and $37^{\circ} \mathrm{C}$ ). Hence, condition of $37^{\circ} \mathrm{C}$ and $24 \mathrm{~h}$ was used as an appropriate condition for IgG coating for subsequent experiments. Zeta potential of uncoated FDS-NPs at all ionic strengths was well below $-30 \mathrm{mV}$ indicating that FDS-NPs were well dispersed and stable when ionic strength was 0.167 . The zeta potentials of IgG coated FDS-NPs at all ion concentrations were about $-40 \mathrm{mV}$ and slightly decreased when ionic strength increased, which means that FDS-NPs are, moderately to well, dispersed in all conditions. Tests with bacteria reveal that IgG coated FDS-NPs tended to create aggregates in the detection of target bacteria; the detection of E. coli O157:H7 on glass slide was successful. FDS-NPs could attach onto target organism and give distinctively bright color. The authors conclude that the detection of bacteria with FDS-NPs is very promising though at low levels of the cells due to their ability of amplify light signal in the detection step. Capture efficiency at $0.2 \mathrm{mg} / \mathrm{mL}$ FDS-NPs 
and 60 min incubation was $87.31 \%$. IgG coated FDS-NPs had low cross-reaction to nontarget bacteria despite using polyclonal antibody with affinity purification.

The paper "Fabrication, characterization, and antimicrobial activity, evaluation of low silver concentrations in silverdoped hydroxyapatite nanoparticles" presented by A. Costescu et al. aims to evaluate $\mathrm{Ca}_{10-x} \mathrm{Ag}_{x}\left(\mathrm{PO}_{4}\right)_{6}(\mathrm{OH})_{2}$ nanoparticles (Ag:Hap-NPs) for their antibacterial and antifungal activities, for $x_{\mathrm{Ag}}=0.02,0.05,0.07$, and 0.10. X-ray diffraction confirms the formation of pure single nanocrystalline HAp phase, which demonstrates that $\mathrm{Ag}^{+}$ions have been successfully substituted $\mathrm{Ca}^{2+}$ ions without affecting its crystal structure. TEM observations reveal uniform ellipsoidal morphology with sizes in the range $5-15 \mathrm{~nm}$ and the rings of selected area electron diffraction (SAED) pattern can be indexed with Hap reflections. Both FTIR and Raman spectra clearly reveal the presence of various vibrational modes corresponding to phosphate and hydroxyl groups. Chemical analysis confirms that $\mathrm{Ca} / \mathrm{P}$ and $(\mathrm{Ca}+\mathrm{Ag}) / \mathrm{P}$ atomic ratios are close to the stoichiometric value of Hap, that is, 1.67. The antimicrobial activity of Ag:HAp $\left(0 \leq x_{\mathrm{Ag}} \leq 0.1\right) \mathrm{NPs}$ was tested using strains belonging to the most commonly encountered pathogens: E. coli ATCC 25922, E. coli 714, K. pneumoniae 2968, B. subtilis, and C. krusei 963 . The results indicate that low Ag concentrations are required to be effective against Gram-negative bacterial and fungal strains and that the intensity of the antimicrobial effect against these strains increases with $\mathrm{Ag}$ concentration. Only high Ag:HAp concentration of $5 \mathrm{mg} / \mathrm{mL}$ was effective against the Gram-positive bacterial strains. The authors concluded that the antimicrobial properties of Ag:HAp NPs support their potential use for various medical applications, for example, textile dressings, orthopedic and dental prostheses, or implants with improved resistance to microbial colonization.

J. Moreno presented a paper titled "Electrodeposition, characterization, and corrosion stability of nanostructured anodic oxides on new Ti-15Zr-5Nb alloy surface." A new Ti$15 \mathrm{Zr}-5 \mathrm{Nb}$ alloy was elaborated by vacuum, cold-crucible, semilevitation melting method. A continuous nanostructured layer of protective $\mathrm{TiO}_{2}$ oxide was deposited galvanostatic anodization in 0.3 and $1 \mathrm{M} \mathrm{H}_{3} \mathrm{PO}_{4}$ (orthophosphoric acid) solution, where three processing parameters were varied: the solution concentration, current density, and time. SEM observations of $0.3 \mathrm{M} \mathrm{H}_{3} \mathrm{PO}_{4}$ show a layer having two hierarchical features: hill-like protuberances with a typical width between 5 and $10 \mu \mathrm{m}$ and nanotube-like porosity with diameters in the one to a few hundred manometers. For $1 \mathrm{M} \mathrm{H}_{3} \mathrm{PO}_{4}$ showed the deposition of loose aggregates of submicron sized particles on top of a porous layer. Raman spectrum clearly shows the existence $\mathrm{TiO}_{2}$ anatase-like structure, indicated by the existence of a strong band at $149 \mathrm{~cm}^{-1}$. There was no evidence of the presence of the main characteristic vibrational band of $\mathrm{PO}_{4}{ }^{3-}$ group. Furthermore, FT-IR spectrum shows two broad bands which were attributed to the $v_{3}$ bending mode of the $\mathrm{P}-\mathrm{O}-\mathrm{P}$ bonds in phospho-titanate glass oxide, due to the incorporation of $\mathrm{P}^{5+}$ ions into the oxide coating. Cyclic potentiodynamic curves make evident a much improved behaviour of the anodized alloy than that of the bare one, due to the formation of nanostructured layer that improves the protective properties of the native passive film by its thickening. Electrochemical impedance spectroscopy (EIS) spectra revealed a better capacitive behaviour and a more insulating protective film. This was attributed to the nanostructured film, which is formed by two layers: an inner, barrier layer that assures the alloy very good anticorrosive resistance, and an outer, porous layer that can provide the good conditions for the bone cell adhesion. Corrosion and ion release rates have lower values showing a better resistance to corrosion and implicitly a more reduced quantity of ions released in biofluid, namely, a lower toxicity of the anodized alloy. The open circuit potentials for the nanostructured alloy shifted to more positive values in time, indicating the increase of its protective layer thickness. The open circuit potential gradients have very low values that cannot generate galvanic corrosion. The obtained material satisfies the most important requirements of an implant material. Its biocompatibility and anticorrosive properties were enhanced by $\mathrm{TiO}_{2}$ oxide which incorporated phosphorus as phospho-titanate $\left(\mathrm{P}_{2} \mathrm{O}_{5}{ }^{2-}\right)$ ions, thus increasing the alloy bioactivity. This composition can stimulate the formation of the bone and the porosity can offer a good scaffold for the bone cell attachment.

The paper titled "Retinoic acid decorated albuminchitosan nanoparticles for targeted delivery of doxorubicin hydrochloride in hepatocellular carcinoma" was presented by J. Varshosaz et al. Retinoic acid-chitosan conjugate (RC) copolymer was confirmed by $1 \mathrm{H}$ NMR and FT-IR. Twelve different formulations of RC-albumin NPs loaded with doxorubicin were prepared and their particle size, PdI zeta potential, loading efficiency (\%), and release efficiency within 60 min $\left(\mathrm{RE}_{60 \%}\right)$ were determined. Statistical analysis showed an increase in degree of substitution of retinoic acid in copolymer which led to a decrease in particle size. Zeta potential demonstrates that the NPs dispersion obtained by coacervation method in an aqueous system is a physically stable system. The amount of total RC and albumin was the most important factor affecting the entrapment efficiency while the role of albumin alone was negligible. In vitro drug release shows a fast release profile for doxorubicin was observed in all formulations. Also, increasing the total mass of RC and albumin significantly decreased the RE. Albumin content had low effect on release behaviour of doxorubicin from NPs. Optimization was done using design expert software and $A_{10} B_{1} C_{0.37} R_{2}$ was suggested as the optimum formulation which showed a good particle size of $286 \mathrm{~nm}$, zeta potential of $30.5 \mathrm{mV}$, an acceptable entrapment efficiency of $43.6 \%$, and relatively high release efficiency of $56.17 \%$. TEM analysis shows NPs with spherical and some irregular shapes. Cell proliferation assay shows that $\mathrm{IC}_{50}$ is decreased in both targeted and nontargeted NPS compared to free doxorubicin; retinoic acid targeted NPs had the lowest $\mathrm{IC}_{50}$ and the highest growth inhibitory effect was observed in cells treated with RC-albumin NPs at $0.5 \mu \mathrm{g} / \mathrm{mL}$. However, the cell survival percentage shows an increase in doxorubicin loaded RC-albumin NPs treated group at $1 \mu \mathrm{g} / \mathrm{mL}$ concentration. Finally, Cellular uptake study reveals uptake of NPS in HepG2 cells. 
Although the issue covered broad topics related to the biomedical applications of NPs, the presented papers discussed some exciting subjects within the scope of Journal of Nanomaterials, which will be beneficial for the readers and scientists.

\section{Acknowledgments}

The editors would like to express their deep appreciation to the authors for their excellent contributions and patience during the reviewing process. Additionally, all reviewers are acknowledged for their great efforts and precious time in assisting us reviewing the papers and their fruitful comments and recommendation for the authors, which improve the quality and depth of the papers.

M. Bououdina

S. Rashdan

J. L. Bobet

Y. Ichiyanagi 

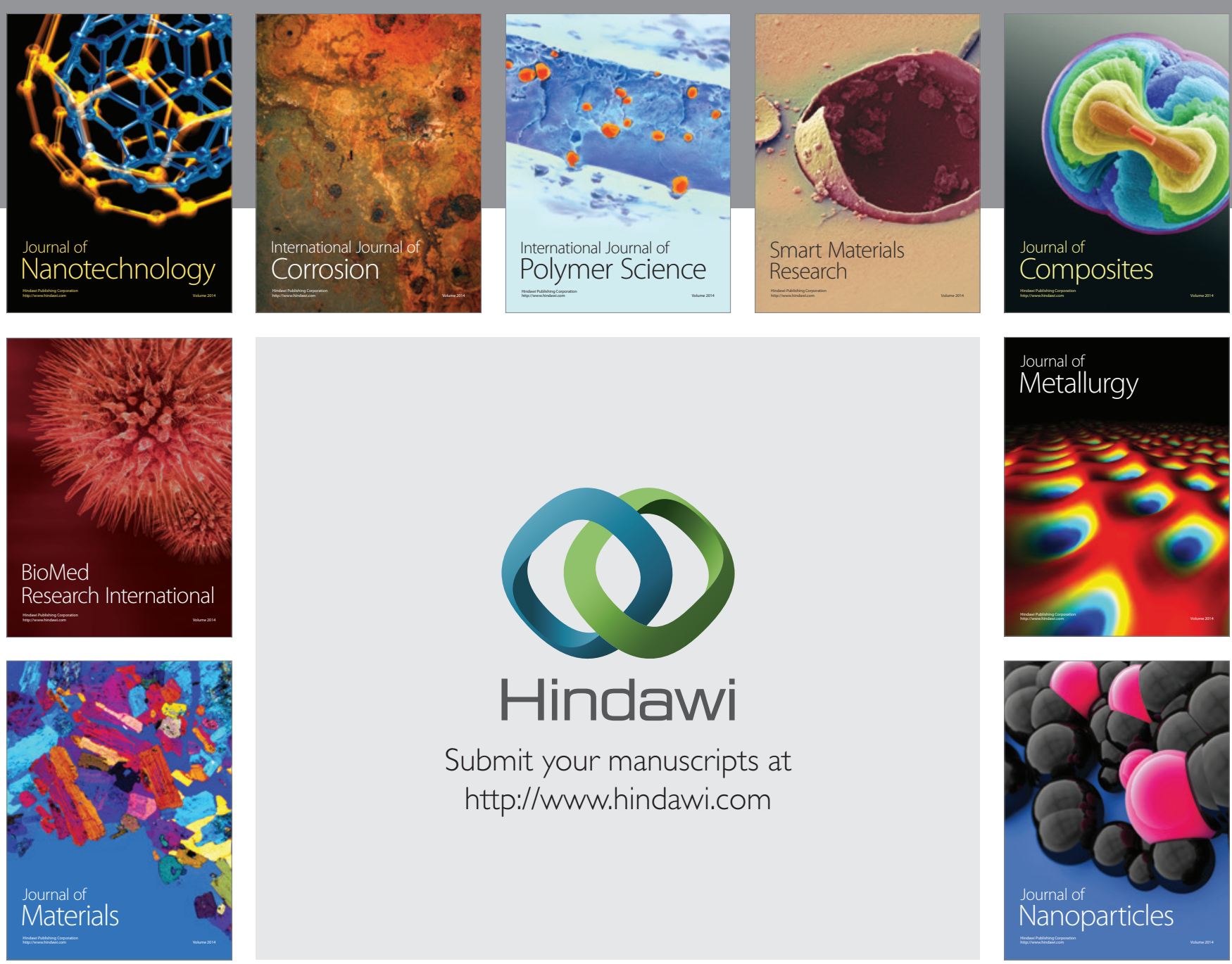

Submit your manuscripts at http://www.hindawi.com
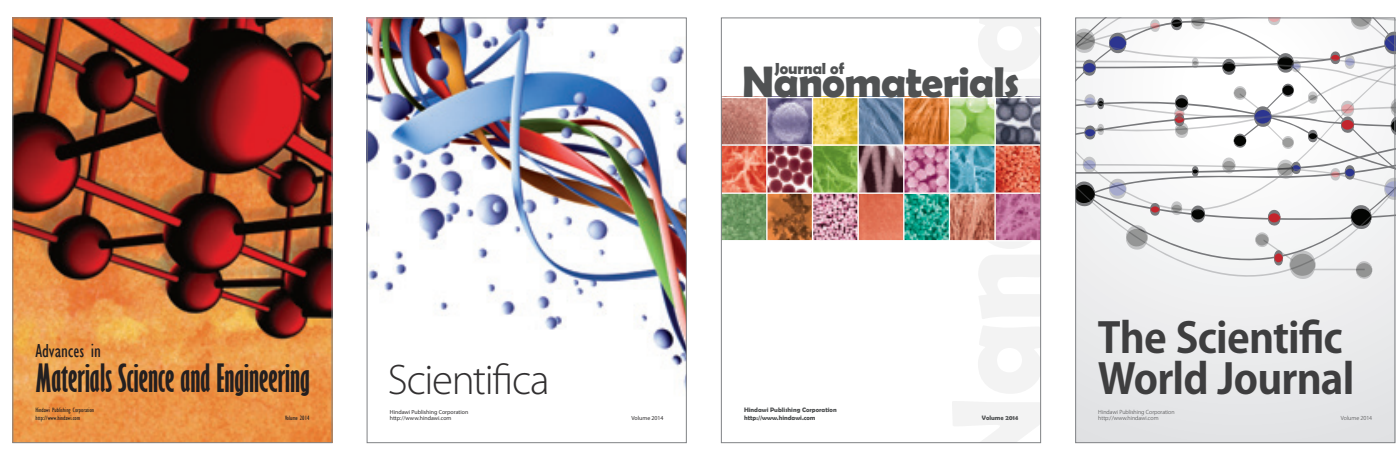

\section{The Scientific World Journal}
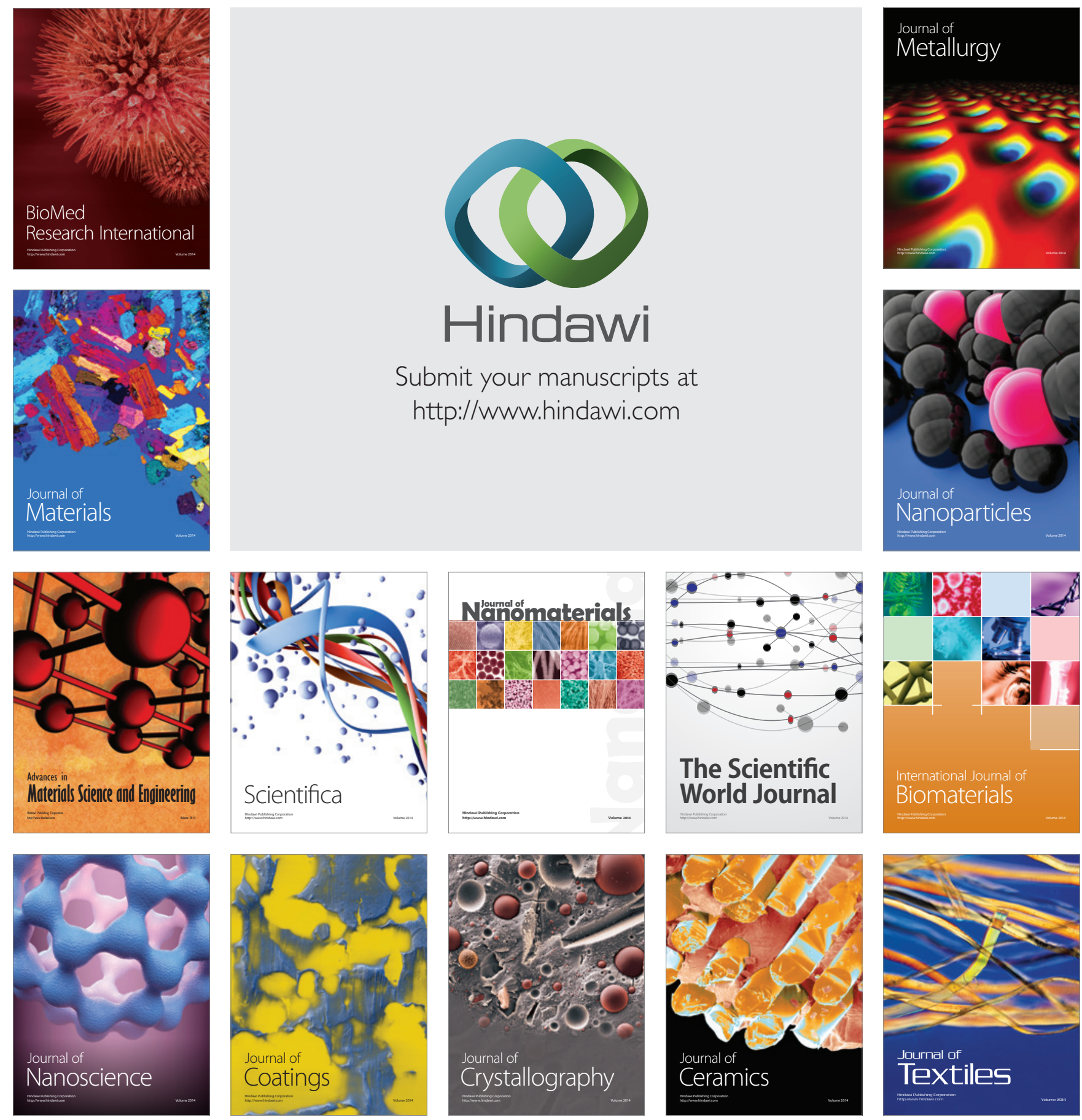